\title{
eJRIEPS
}

Ejournal de la recherche sur l'intervention en éducation physique et sport

$44 \mid 2019$

Varia

\section{Analyse des pratiques d'enseignement des sports de combat à l'université en France : deux études de cas de formateurs en STAPS}

Analysis of combat sports teaching practices in the university: two case studies of professors in STAPS

Mariana S.P. Gomes, Marie France Carnus et André Terrisse

\section{(2) OpenEdition}

Journals

Édition électronique

URL : http://journals.openedition.org/ejrieps/405

DOI : 10.4000 /ejrieps.405

ISSN : 2105-0821

Éditeur

ELLIADD

Référence électronique

Mariana S.P. Gomes, Marie France Carnus et André Terrisse, « Analyse des pratiques d'enseignement des sports de combat à l'université en France : deux études de cas de formateurs en STAPS », eJRIEPS [En ligne], 44 | 2019, mis en ligne le 01 janvier 2019, consulté le 01 août 2019. URL : http:// journals.openedition.org/ejrieps/405; DOI : 10.4000/ejrieps.405

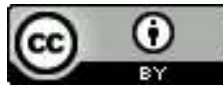

La revue eJRIEPS est mise à disposition selon les termes de la Creative Commons Attribution 4.0 International License. 
eJRIEPS 44 janvier 2019

\section{Analyse des pratiques d'enseignement des sports de combat à l'université en France : deux études de cas de formateurs en STAPS}

Mariana S.P. Gomes 1, 2, Marie France Carnus 2, André Terrisse 2

1. Laboratoire d'Education Physique Adaptée, Faculté d'Education Physique - Université de Campinas, Brésil

2. UMR-EFTS, - Université Toulouse Jean Jaurès, France

\section{Résumé}

Cette recherche se propose d'analyser deux enseignements de sports de combat différents, l'escrime et la savate boxe française (SBF), destinés aux étudiants de la même Unité de Formation et de Recherche en Sciences et Techniques des Activités Physiques et Sportives (UFRSTAPS), pour en décrire la logique différentielle. La question de recherche porte sur la nature des différences constatées en terme de traitement de ces activités du point de vue de deux enseignants singuliers par leur passé de sportif et leur formation. Certes, ces deux activités sont différentes par leur histoire et leur contenu, bien que faisant partie toutes les deux de la catégorie des sports de combat. Ce que la recherche tente de mettre en évidence porte davantage sur les raisons de cette différence de traitement, soit leur expérience en tant que pratiquant ou en tant qu'enseignant, ce que mettent en exergue les travaux de l'Equipe de recherche en Didactique Clinique de I'Education Physique et Sportive (EDiC) et son cadre théorique de la didactique clinique (Carnus \& Terrisse, 2013).

Pour y parvenir, nous avons tenté de définir le "savoir combattre " que le professeur peut transmettre dans ces activités, en utilisant des catégories d'analyse de la pratique d'enseignement des sports de combat qui nous permettent de différencier les formes didactiques, comme le processus de dévolution et certaines variables didactiques, le rapport de force, la répétition décontextualisée du rapport de force et l'arbitrage. Nous avons alors observé que la différence de formation de ces deux enseignants dans le domaine sportif et à l'Université a pesé sur les raisons d'un enseignement différent de Jean (Escrime) et de Roger (SBF). Cette recherche met en évidence le fait que chaque professeur fait ses choix en fonction de ce qu'il a vécu, soit comme pratiquant, soit comme étudiant en STAPS, soit encore comme professeur.

Mots clés : didactique clinique, sports de combat, vécu sportif, pratiques d'enseignement 


\section{eJRIEPS 44 janvier 2019}

\section{Introduction}

L'enseignement des sports de combat est, comme la plupart des Activités Physiques, Sportives et Artistiques (APSA), influencé par les traditions et les valeurs que leur accorde chaque enseignant en fonction de la conception qu'il s'est construite (Terrisse, 2000). En effet, dans ces activités, le professeur se présente souvent comme un "seisei", nom donné au maître, ou bien se situe plutôt comme un sportif qui a plusieurs années d'expérience et de pratique (Gomes, Morato, Duarte, Almeida, 2010). Cette particularité nous amène à élaborer un cadre d'analyse des pratiques d'enseignement qui tienne compte de cette spécificité.

Ainsi, en rapport avec l'analyse de l'enseignement des sports de combat telle que la propose Terrisse (1991), Gomes (2008) a identifié les éléments qui déterminent la logique du savoir combattre, soit ce qu'elle nomme les principes conditionnels que sont le contact intentionnel, l'action simultanée attaque/défense, l'incertitude, la cible que constitue l'adversaire et les règles. Ces déterminants constitutifs de la pratique montrent que l'on peut dégager une logique interne qui pourrait se nommer le savoir combattre (Terrisse, Quesada, Hiegel, Sauvegrain, 1995) et qui peut être utilisée comme cadre d'analyse de la pratique d'enseignement, dépassant ainsi le contexte institutionnel (école, club ou loisir) où le sport de combat est enseigné.

D'autre part, plusieurs auteurs montrent que l'enseignement entraîne une transformation du savoir quand il est transmis par un enseignant, ce que Chevallard nomme la transposition didactique interne (Chevallard, 1985). En effet, pour être enseignés, les savoirs doivent être rendus enseignables (Reuter, 2007) comme Verret l'a montré lorsqu'il a étudié les conditions de transmissibilté des savoirs (Verret, 1975). Cette transformation rend compte de la transformation d'un savoir «déjà-là », produit, entre autres de l'expérience du pratiquant, voire de l'enseignant, à un savoir à enseigner à des étudiants, puis à un savoir réellement enseigné, transformation qui est l'objet de notre recherche.

En effet, celle-ci se réfère à ce processus de transposition didactique interne dans la mesure où elle tente de rendre compte des savoirs que choisissent de transmettre deux professeurs dans une même institution et pour les mêmes étudiants. Compte tenu de leurs différences de formation, il peut être possible d'observer deux types de savoirs distincts non exclusifs l'un de l'autre: soit des contenus en référence à leur propre expérience de "combattant", comme témoins de leur mémoire, soit des contenus orientés et traités pour être utilisés lorsque ces étudiants seront eux-mêmes en situation de les transmettre. En fonction des catégories d'analyse déjà évoquées, le chercheur tentera alors de répondre à 
eJRIEPS 44 janvier 2019

cette question de l'origine des savoirs délivrés par ces deux enseignants et de la nature de leur différence.

\section{Options théoriques et revue de questions}

2.1 . Une recherche en didactique clinique des sports de combat.

Cette étude se situe dans le champ de la didactique clinique de l'EPS qui met au cœur de ses analyses le sujet enseignant en tant qu'être singulier, assujetti et divisé (Carnus, 2004; Carnus, Terrisse, 2013). Dans cette orientation théorique, les choix didactiques des enseignants sont doublement influencés par des facteurs externes relevant des contextes et des institutions et des facteurs internes, propres aux sujets, à leur histoire, à leur « déjàlà ». Centré sur le suivi des enjeux de savoir à travers la logique des sujets engagés dans la relation didactique (Carnus, 2009a), ce positionnement autorise une autre lecture du fonctionnement didactique guidée par l'histoire et la singularité des sujets construites au carrefour de l'intime et du public (Carnus, 2009b).

Cette approche nécessite que soient questionnés un certain nombre de concepts, notamment celui de transposition didactique qui, importé dans le champ didactique clinique, est revisité par la prise en compte de la dimension du sujet. Nous utilisons aujourd'hui le terme de conversion didactique, soit « une production de symptômes témoignant d'une construction psychique du sujet enseignant élaborée au cours de son histoire » (Buznic, 2009 ; Carnus \& Terrisse, 2013, p 141). Le savoir à enseigner, qui renvoie à l'intention didactique, relève de trois sources d'influences: interne, liée à l'expérience et à l'expertise de l'enseignant ; institutionnelle liée aux programmes et à leur interprétation par l'enseignant ou le formateur; sociale ou culturelle, en lien avec la pratique sociale de référence et sa logique interne.

Nous allons présenter dans ce chapitre trois recherches qui posent cette question de conversion didactique pour en comprendre les enjeux.

Ainsi, Brossais et Terrisse (2009) ont ils étudié le poids de l'institution sportive, et donc de la formation antérieure, d'un enseignant d'EPS débutant expert en judo. La recherche indique qu'il transmet ce qu'il a appris en tant que judoka et qu'il reproduit la même structure d'apprentissage que la Fédération Française de Judo lui a apprise, sans considérer les stratégies nécessaires à l'opposition, dont les adolescents sont pourtant friands. Cet enseignant dira d'ailleurs : "quel que soit le milieu, je l'aurais enseigné de cette manière" (Brossais, Terrisse, 2009, p. 131). Car un combat se définit surtout grâce à l'incertitude de son issue qui est due au rapport de force entre les combattants ce qui 


\section{eJRIEPS 44 janvier 2019}

semble éloigné des situations décontextualisées (Chevallard, 1985) et des répétitions de gestes sans rapport avec le contexte de l'opposition que peuvent transmettre les enseignants de sport de combat, même si la transmission des techniques à travers les formes de corps, s'avère indispensable à connaître par les collégiens. Dans ce cas apparaît l'importance de la formation pratique antérieure de l'enseignant dans le choix de ses contenus de formation, ce qui justifie l'utilisation du terme de conversion didactique.

Toutefois, la transmission du savoir peut subir d'autres influences, notamment institutionnelles. Pour cette raison, Heuser (2009) a observé des écarts entre le savoir enseigné en EPS et le savoir de référence chez un spécialiste de karaté. II relate que Nicolas (le cas étudié) avait eu une expérience soutenue comme athlète de compétition avec des combats « durs » mais, lorsqu'il enseignait, il ne mobilisait pas ce savoir de référence pour le modifier en fonction du niveau de la classe avec laquelle il travaillait, mais aussi des valeurs dispensées dans le milieu scolaire (Heuser, 2009). Cette étude de cas permet de comprendre que la référence de l'enseignant n'est pas toujours celle du pratiquant, compte tenu des normes et des valeurs que véhicule l'institution scolaire et auxquelles l'enseignant ne peut pas déroger.

Enfin, dans le cadre d'une recherche sur les savoirs appris par les élèves, Sauvegrain (2001), expose le cas de Florent qui, dans un cycle de lutte à l'école, ne reproduit pas ce que l'enseignant lui a transmis. II invente une technique pour résoudre le problème qu'il rencontre dans l'activité d'opposition avec un autre élève. Sauvegrain observe que l'enseignant, expert en lutte, a choisi un contenu que Florent ne reproduit pas. Par contre, Florent a compris le sens de la lutte selon ses propres nécessités. Terrisse (2009) signale que, dans ce cas, le chercheur doit rendre compte du rapport au savoir construit par l'élève pour comprendre les conditions de sa transformation et ainsi mettre en évidence un savoir par la pratique, qui est une forme de conversion, adaptée au contexte de l'opposition, mais effectuée par l'élève lui-même.

C'est donc au carrefour de ces influences que s'élabore le processus de conversion didactique relative au savoir enseigné. Cette redéfinition permet de mieux comprendre les variabilités inter individuelles souvent observées dans l'analyse des pratiques enseignantes (Bru, 1991). Nous sortons ici d'une vision linéaire de la transposition didactique et nous nous focalisons sur la question des écarts, notamment entre savoir à enseigner et savoir effectivement enseigné, et même, dans le dernier exemple, entre savoir enseigné et savoir appris. 


\section{eJRIEPS 44 janvier 2019}

2. 2. Une recherche sur la nature du savoir combattre

Pour pouvoir analyser ces processus de transmission à l'aide du concept de conversion didactique dans le cadre théorique de la didactique clinique, nous analyserons le savoir combattre et les possibilités d'enseignement qui s'offrent à l'enseignant dès qu'il s'approprie ce savoir.

Figueiredo (2009) a décrit les activités de combat comme des activités où le corps de l'autre est l'objet et le but de l'enjeu des actions intentionnelles de combat. Green \& Svinth (2010, p.19) comprennent ces activités comme des «systèmes qui combinent les éléments physiques du combat avec stratégie, philosophie, tradition et d'autres caractéristiques qui les différencient de la pure réaction ».

Terrisse (1991) envisage qu'une pensée dialectique puisse permettre au professeur la gestion de l'opposition et de la coopération, dans la mesure où il peut utiliser un rapport de force pour enseigner ces objectifs, et où il peut réduire l'imprévisibilité des situations de combat par la coopération notamment.

Le savoir combattre pour Terrisse et al. (1995, p. 27) peut être représenté par une intention stratégique engagée dans une «pleine opposition » qui suscite « la nécessité de réduire l'incertitude pour faciliter l'apprentissage et en même temps conserver les phases de confrontation afin de ne pas perdre le sens de l'activité ».

La stratégie, pour Loizon, Margnes et Terrisse (2004, p. 69) est conçue comme « un plan d'action organisé dans un but précis » alors que la tactique « est la mise en œuvre de ce plan, l'adaptation de celui-ci aux contraintes de la situation ».

Pour Terrisse et al (1995, p. 27), l'intention stratégique vise à résoudre les problèmes d'un combat. Au-delà du concept de savoir combattre, vers une orientation plus clinique, Terrisse (2000) a proposé trois types de savoirs qui caractérisent, d'après lui la nature des savoirs en sports de combat à partir des travaux réalisés par Sauvegrain sur l'enseignement de la lutte en 1998 (Terrisse et Sauvegrain, 1998) :

- "Un savoir de la pratique" que les auteurs annoncent comme étant le produit de l'histoire de la pratique, "des règles sociales qui la régissent et des valeurs qui y sont attachées". ॥ y a en judo, un ensemble de valeurs, traditions et conceptions qui font partie d'un domaine social, de la communauté du judo, qui peuvent se manifester à travers la culture et le contexte de pratique propre au judo.

- "Un savoir pour la pratique", qui est le fruit d'un travail de mise en forme par les entraîneurs et les enseignants de contenus, de conseils, de stratégies et de techniques. 


\section{eJRIEPS 44 janvier 2019}

C'est le savoir qui est élaboré et qui est mis en pratique par le professeur pendant ses cours. II peut correspondre aux préconisations des professeurs (Terrisse, 2000).

- "Un savoir par la pratique", qui est le produit de la confrontation personnelle à autrui et qui ne s'acquiert que dans l'épreuve de combat, que Terrisse nomme "le savoir y faire. » (Terrisse, 2000, p.105). C'est l'intention tactique qui émerge de la pratique des étudiants, comme une réponse aux problèmes proposés par le professeur, mais aussi par les adversaires pendant la situation d'opposition.

Dans la mesure où notre projet de recherche est d'analyser des pratiques d'enseignement des sports de combat à l'Université à partir de cette notion de savoir combattre, nous utiliserons ces différences entre le savoir de la pratique, le savoir pour la pratique et le savoir par la pratique, à travers des catégories d'analyse qui permettront d'observer et de caractériser cet enseignement. Alors que le savoir de la pratique ne semble pas subir beaucoup de modifications car le professeur transmet souvent ce qu'il a appris en tant que pratiquant sous forme de théorie, de traditions et de valeurs, le savoir pour la pratique exige du professeur une transformation des savoirs sous forme de contenus didactiques afin de transmettre les contenus les plus utiles aux étudiants. Enfin, le savoir par la pratique s'acquiert par l'activité de combat pour chacun des étudiants et il se trouve donc difficile à observer.

\section{Questions de recherche}

Notre sentiment, fruit de notre formation en combat, est qu'un professeur à l'Université ne va pas seulement transmettre des savoirs sportifs tels qu'il les a vécus lui-même, bien que ce soit le socle de ce qu'il peut transmettre, mais qu'il va tenter de les traiter en fonction des objectifs de formation qu'il se donne ou qui sont exigés par l'Institution pour laquelle il travaille. Nos questions de recherche deviennent alors :

Quelle est la nature du savoir combattre pour chacun des deux professeurs observés ? Y a-t-il des différences de transmission de ce savoir selon les professeurs en fonction de leur expérience, de leur propre formation et/ou des objectifs qu'ils se fixent?

Nous faisons l'hypothèse que la transmission du savoir combattre passe par un certain nombre de notions et de pratiques propres à chaque enseignant. Notions et pratiques seront considérées par les enseignants comme fondamentales à acquérir par les étudiants pour combattre, ce que nous allons tenter d'extraire de nos observations.

Nous allons analyser l'enseignement des sports de combat dans ce contexte institutionnel de deux professeurs qui sont dans la même institution universitaire en utilisant les 


\section{eJRIEPS 44 janvier 2019}

catégories d'analyseurs qui représentent les caractéristiques spécifiques de la didactique des sports de combat pour en déterminer la logique, que nous envisageons différente du fait que ce sont deux enseignants singuliers, avec des histoires de pratiquants différentes, celles de la SBF et de l'escrime.

\section{Méthodologie}

Cette recherche qualitative vise à observer, comprendre et interpréter le phénomène étudié en considérant surtout son essence (Thomas \& Nelson, 2002). Elle prend les pratiques d'enseignement comme objet d'analyse pour en extraire la nature du savoir.

Selon Terrisse et Sauvegrain (1998), deux difficultés peuvent se présenter dans la recherche qualitative : d'une part, préciser les raisons pour lesquelles sont recueillies certaines données et d'autre part, affirmer les options théoriques justifiant cette analyse et son interprétation. Dans ce cas de recherche didactique clinique, l'entretien est l'outil prioritairement utilisé en considérant "qu'un dire permet au sujet de soutenir les motifs de sa pratique et d'énoncer le bénéfice qu'il en retire, car toute activité humaine devrait avoir un sens, même s'il est non su" (Terrisse \& Sauvegrain 1998, p.99).

Nous utilisons alors, la méthodologie temporelle de la didactique clinique : le déjà-là, l'épreuve et l'après-coup (Terrisse, Carnus, 2009).

\section{1. Les sujets}

Cette étude fait partie d'un projet de recherche de doctorat qui se propose d'analyser et de comparer les pratiques d'enseignement en Sports de Combat à l'Université dans deux pays différents : la France et le Brésil. Dans cet article, nous n'analyserons que les pratiques d'enseignement en France et plus particulièrement une séance de deux professeurs enseignant dans un UFRSTAPS ${ }^{1}$. Ils travaillent dans la même université et leurs cours font partie de la première année (tronc commun) en travaux pratiques (TP), où il y a entre 15 et 20 étudiants par groupe. Les étudiants sont tirés au sort pour pratiquer les trois types de sports de combat offerts par cette Université (Escrime, SBF et Judo). Ces étudiants viennent du lycée, où d'après nos informations, ils ont eu peu d'expérience en sports de combat dans les cours d'Education Physique et Sportive (EPS).

Pour des raisons d'anonymat, nous nommerons le premier professeur, enseignant la SBF, Roger et le deuxième professeur, enseignant l'escrime, Jean.

\footnotetext{
${ }^{1}$ Le chercheur a choisi de travailler avec ces deux enseignants à partir d'une information donnée par l'un d'entre eux dans une réunion de travail de l'équipe de recherche qui a dirigé ces travaux. II a eu connaissance du parcours des deux enseignants grâce à des entretiens ante qui ont pour objectif de connaître les origines de leur formation et leur projet d'intervention.
} 


\section{eJRIEPS 44 janvier 2019}

Roger a connu la SBF à la fin de ses études à l'UFRSTAPS. II n'a pas été un combattant de haut niveau dans cette discipline sportive, mais il a entraîné plusieurs « tireurs » de boxe française et il a obtenu, dans ce contexte et avec ses athlètes, des titres nationaux. Aujourd'hui, il est professeur de SBF dans l'institution où il a suivi sa formation.

A contrario, Jean a découvert l'escrime quand il avait 9 ans. II a vécu le sport dans un contexte scolaire "sport-étude" et en club. Après le bac, il a été intégré dans l'équipe de France d'escrime (spécialité sabre), II a effectué sa formation en sport en même temps que celle de professeur d'EPS. Compétiteur de haut niveau jusqu'en 2004, il a été champion olympique de sabre par équipe. Actuellement, il est professeur de sport et maître d'armes.

4. 2. Le recueil et le traitement des données

4. 2. 1. Entretien ante-séance (accès au déjà là)

Selon cette méthodologie, qui privilégie la prise en compte temporelle du déroulement des séances, nous avons débuté le recueil des données par l'entretien ante séance avec chaque professeur afin de connaître leur déjà-la particulièrement focalisé sur leurs intentions didactiques, leurs objectifs et leurs prévisions d'intervention. Ce déjà-là constitue, pour nous, «le savoir lié au processus historique de son mode de construction dont se déduit la logique de l'activité » (Terrisse, 1994). Ces entretiens ont été fidèlement retranscrits et analysés selon une analyse de contenu telle que la décrit Bardin (1998) : lecture flottante puis reconstruction du cas à partir des éléments les plus saillants au regard des questions de recherche afin de substituer à l'interprétation intuitive une interprétation construite au moyen d'une inférence. A la lecture de ces entretiens, nous avons repéré et souligné les points les plus importants de cette prévision pour nous préparer à l'étape suivante, c'est-à-dire, à l'observation et à l'enregistrement vidéo des séances, en tenant compte des indications de ces deux professeurs, ce qui constitue la deuxième phase de ce type de recueil de données que nous nommons l'épreuve.

\section{2. 2. Enregistrements Vidéo au cours de l'épreuve}

Lors de ce second temps de la méthodologie, nous avons choisi de filmer et d'enregistrer les tours de paroles entre le professeur et les étudiants au cours d'une séance afin de chaque enseignant pour suivre le déroulement de leur intention didactique. II s'agit de la première séance d'un total de trois, qui font partie du projet de doctorat. Nous avons enregistré les trois dernières séances pratiques avant les assauts, qui constituent le moment d'évaluation des acquisitions. Pour cet article, nous n'utiliserons que les données filmées et enregistrées de la première séance, car la richesse de données observées a été 


\section{eJRIEPS 44 janvier 2019}

complétée par les entretiens ante et d'après-coup. Cette observation a permis de révéler le type de savoir enseigné en SBF et Escrime, par ces deux professeurs, dans cette université

Pour ce faire, nous avons utilisé une vidéo caméra digitale et un micro-cravate attaché au professeur, pour bien l'entendre au moment de l'enregistrement afin de faciliter l'écoute et la transcription de ses interventions. La camera était positionnée de manière à filmer tous les étudiants et surtout la pratique d'enseignement du professeur.

Avant de commencer leur cours, ces enseignants expliquent les objectifs de la séance, leur plan de travail et ce qu'ils attendent des étudiants. Après le cours, chaque professeur vient relater sa séance au chercheur en la comparant à ses prévisions, sous la forme d'entretiens post séance. Ces entretiens permettent de savoir à quel moment et pour quelle raison ils ont changé leur planification, par exemple. Cela nous a permis d'observer des écarts entre leur projet et leur pratique réelle, soit les savoirs réellement transmis au cours de sa séance, ce qu'ont mis en évidence les travaux de l'EDiC (Carnus, 2001, Buznic, 2009; Ben Jomaa, 2009, Heuser, 2009). Ces écarts sont essentiels à prendre en compte pour mettre en évidence ce que révèle le cadre théorique de l'EDiC et de mieux comprendre l'intérêt de cette option de recherche qui ne postule pas la continuité, mais la rupture entre les intentions didactiques et l'observation des faits réels de l'enseignant « in situ ».

\section{2. 3. Analyse des données}

Dès l'observation in situ terminée, nous avons étudié les vidéos selon les catégories d'analyse retenues, qui seront développées dans le point 4.3.

Dans un premier temps, chaque vidéo a été transcrite et nous avons fait ce que Bardin (1998) a appelé « l'inférence individuelle » (le chercheur essaie d'extraire les contenus latents de chaque « verbatim »). Dans un second temps, nous avons mis en évidence les points communs et les divergences dans les discours et les pratiques selon les catégories d'analyse et le cadre théorique.

Cette première analyse a contribué à la construction des entretiens d'après-coup ${ }^{2}$ qui vont permettre de revenir sur les observations pour éclairer ce que le chercheur n'a pu expliquer en visionnant la vidéo et ainsi confronter les intentions initiales et le discours des professeurs avec leur pratique auprès des étudiants.

\footnotetext{
${ }^{2}$ En didactique clinique, l'entretien d'après coup permet de revenir sur le déroulé de la séance et de mettre en perspectives les intentions des enseignants avec la réalité de la séance observée.
} 


\section{eJRIEPS 44 janvier 2019}

\section{3. Entretien d'après-coup}

Nous terminons le recueil de données par un entretien d'après-coup qui permet aux enseignants de revenir sur leurs pratiques et d'analyser leurs choix d'enseignement, dans le but d'en extraire les raisons. Ce troisième temps de la méthodologie permet aux enseignants de reconstruire le sens de leurs choix par le remaniement des traces mnésiques de l'expérience. Cette reconstruction, confrontée aux hypothèses ou intuitions du chercheur, permet d'accéder aux causes des choix et à la part insu dans l'élaboration des contenus d'enseignement.

4. 4. Les catégories d'analyse de la pratique d'enseignement des sports de combat Pour différencier les aspects didactiques des aspects pratiques chez les enseignants, nous avons choisi des catégories d'analyse déjà utilisées dans d'autres travaux sur l'enseignement de l'EPS et l'enseignement des sports de combat et qui sont particulièrement heuristiques (Loizon, Margnes, Terrisse, 2004; Gomes et al. 2010). En étudiant les discours des professeurs, les séances observés et l'entretien d'après-coup, nous avons essayé d'extraire les contenus qui répondent aux objectifs de la recherche, selon les catégories, qui caractérisent le traitement didactique de chaque professeur analysé.

\section{4. 1. Le processus de dévolution : analyseur des pratiques}

Selon Brousseau (1998) «la dévolution désigne l'ensemble des actions de l'enseignant visant à rendre l'élève responsable de la résolution d'un problème » (p. 303). Dans ce cas, les étudiants prennent la responsabilité de la tâche (Reuter, 2007). Cette catégorie d'analyse permet au chercheur de savoir si l'enseignant a envisagé la prise en charge du savoir par les étudiants au travers la nature de tâches qu'il met en oeuvre. Pour cette raison, les indicateurs pour observer le processus de dévolution sont à référer à la différence que fait Famose entre tâches définies et tâches non ou semi définies (Famose, 1983).

Selon ces tâches différentes, nous analyserons si l'étudiant doit prendre des initiatives visant à s'approprier le savoir comme réduire la distance de combat pour toucher l'adversaire ou au contraire, attendre l'attaque pour mieux riposter.

\section{4. 2. Les variables didactiques}

Loizon, Margnes, Terrisse (2004) définissent, après Brousseau, le concept de variables didactiques comme « les éléments constitutifs des situations d'enseignement identifiables sous forme de contraintes sur lesquelles l'enseignant peut agir en fixant les valeurs afin de 


\section{eJRIEPS 44 janvier 2019}

conduire l'élève vers un apprentissage donné » $(p .70)^{3}$. Cet outil nous permet de reconnaître un traitement didactique de la part de ces professeurs lorsqu'ils manipulent ces variables afin de faciliter l'apprentissage des étudiants, comme la modification de la distance de garde, la vitesse de réaction de l'attaqué ou le changement d'adversaire. Nous intégrons également dans cette catégorie le rapport de force, la répétition décontextualisée et l'arbitrage.

\section{4. 2. 1. Le rapport de force}

On peut observer dans certains manuels d'enseignement des sports de combat, en escrime et en SBF, une option de traitement décontextualisée des savoirs sous la forme de coopération (comme transmettre les Katas, formes de corps répertoriées), ou bien une option d'enseignement plus frontale, en utilisant l'opposition à autrui, ce qui est, d'après la plupart des experts, le savoir fondamental à transmettre. Ainsi, le traitement didactique consiste-t-il à articuler les deux formes de rapport, entre une dialectique entre la coopération et l'opposition ou proposer une forme d'enseignement décontextualisée. Ainsi, la nature du rapport de force devient-il une variable didactique dans la mesure où plusieurs modalités peuvent être envisagées : soit une coopération, soit une opposition entre les deux opposants. Cette différence est fondamentale dans l'enseignement des sports de combat (Terrisse, 1991).

\section{4. 2. 2. La répétition décontextualisée}

Cette modalité correspond à la reproduction des mouvements sous la forme d'une automatisation, ce qui est le contraire d'une forme d'enseignement "dialectique", où est prise en compte la situation d'affrontement, où les rôles ne sont pas définis à l'avance. Cette tendance est une des formes d'enseignement historiquement datée, mais souvent encore utilisée. La répétition est dite décontextualisée dans la mesure où elle est sortie de son contexte d'opposition et elle est ramenée à une coopération, sortie de son contexte de rapport de force.

\section{4. 2. 3 L'arbitrage}

Dans l'observation de séances d'enseignement des sports de combat, nous avons observé que des aspects formels de l'activité, comme les règles d'arbitrage sont quelquefois valorisées par l'enseignant qui met l'accent sur un savoir "réglementaire", savoir nécessaire pour organiser les combats (Margnes, 1996). Ce savoir, fondamental en combat, est plus ou moins valorisé selon les enseignants. S'il est indispensable à la pratique, il peut

\footnotetext{
${ }^{3}$ Entre savoir et variable, il existe une différence notable dans la mesure où l'utilisation d'une variable a, normalement, pour effet de produire du savoir, notamment du « savoir combattre ».
} 


\section{eJRIEPS 44 janvier 2019}

devenir pour certains enseignants un "savoir à savoir" (Chevallard, 1985). La question de l'arbitrage est pertinente comme variable didactique, car proposer aux étudiants d'arbitrer est une question didactique dans la mesure où cette solution permet d'intégrer les normes, les règles et les valeurs de l'APSA et surtout devient un moyen, pour l'enseignant, de donner un sens aux situations qu'il propose. Émettre un jugement sur une confrontation devient alors un moyen d'évaluer in situ les apprentissages visés.

\section{Principaux résultats}

L'analyse des pratiques de chaque professeur a été effectuée à travers les catégories d'analyse décrites ci-dessus, qui révèlent comment les professeurs transmettent le savoir combattre en identifiant si leur démarche mobilise plutôt sur les savoirs de la pratique, pour la pratique ou par la pratique. Ces catégories se configurent sous la forme de caractéristiques didactiques qui peuvent être présentes dans l'enseignement de chaque professeur analysé. Elles nous ont permis d'observer les séances et de voir la différence de traitement didactique chez les deux professeurs observés.

\section{1. Analyses des entretiens ante}

5. 1. 1. Roger en Savate Boxe Française

Pendant l'entretien ante-séance, Roger nous dit que les étudiants vont travailler la technique. Toutefois, il leur demande de faire attention aux éléments surtout tactiques (déplacements, parades, esquives). Quand il laisse les étudiants choisir ce qui est le plus intéressant pour eux, il laisse la technique surgir comme une réponse aux exigences du combat.

\section{1. 2. Jean en Escrime}

Pendant l'entretien ante-séance, Jean annonce que ses objectifs sont de travailler sur les niveaux de défense en escrime pour augmenter le potentiel d'attaque et de défense chez les étudiants. II veut organiser une révision « d'une mise en activation » et automatiser le déplacement, la prise d'information et la coordination des étudiants. II semble que ses objectifs, dans le cas de cette séance, vont plutôt vers la répétition, puisque l'automatisation est directement articulée à la répétition, contextualisée ou non.

5. 2. Analyse des pratiques au temps de l'épreuve

5. 2. 1. Analyse de la pratique en SBF

La séance analysée est une des dernières séances pratiques du cycle du deuxième semestre. Nous l'avons choisi parce que les étudiants ont déjà vécu plusieurs heures dans chaque activité. Cette observation peut rendre compte des écarts entre le discours et 


\section{eJRIEPS 44 janvier 2019}

la pratique du professeur. Selon les objectifs exposés par Roger pendant l'entretien anteséance, son intention est d'insister sur l'introduction de la notion de feintes et de rythme. II veut « retravailler les enchaînements pied-poing-poing-pied et revenir sur l'esquive (rotative et sur place) ». (Entretien ante-séance SBF)

Au début de la séance, Roger demande à un des étudiants de prendre en charge l'échauffement, qui est un échauffement général sans rapport particulier avec ses objectifs. Le professeur revient sur ce qu'il a travaillé la dernière fois et commence les activités en partageant les étudiants en binômes. II les met dans un rapport de force, c'està-dire face à face, dans un mini ring et leur donne quelques consignes pour mettre l'accent sur ce qu'il veut travailler dans le cours, soit l'enchaînement :

"Donc le tireur A attaque, crochet, swing au revers et le tireur B esquive. Je n'ai pas dit "pare ». Vous ne parez que lorsque vous n'avez pas d'autres choix. Donc la priorité, c'est d'éviter de se faire toucher et de pouvoir enchainer le plus vite possible. Donc on enchaîne par un enchainement libre pied poings ou poings pieds de son choix, mais le but, c'est de pouvoir anticiper." (SBF-Séance)

Roger donne des consignes en expliquant les actions à réaliser qu'il souhaite. Par contre, il laisse les étudiants choisir de les utiliser ou pas. II semble vouloir leur faire comprendre que la logique du combat détermine le moment d'utiliser les techniques. Quand il met ses étudiants en opposition, ils sont obligés de trouver le moment d'utiliser les techniques spécifiques.

Toutes les trois ou quatre minutes, il change soit les consignes, soit les rôles, soit les partenaires. Nous observons donc de sa part une manipulation évidente des variables didactiques que sont les rôles ou les adversaires dans le rapport d'opposition. Toute la séance se déroule avec les étudiants en situation semi-définie d'opposition (Famose, 1983) et même s'il donne souvent des explications de nature biomécanique, il précise que c'est pour mieux répondre aux problèmes qu'il leur a posés. Cela semble montrer que pour lui, la technique est au service de la tactique : « le mouvement de la feinte, le début du mouvement est lent, d'accord, et ensuite on l'accélère pour toucher." (SBF-Séance). Nous constatons un entrelacement des savoirs, dans la mesure où le savoir pour la pratique (plutôt technique) se mélange avec le savoir par la pratique (la tactique dans le combat).

Roger insiste d'ailleurs plusieurs fois sur ce savoir important pour lui qu'est la liaison technique/ tactique. II corrige les mouvements selon le comportement de l'adversaire en contexte dans le rapport de force. II fait attention à la technique. Par contre, comme il met 


\section{eJRIEPS 44 janvier 2019}

les étudiants en situation d'opposition, il ne sépare pas les aspects techniques des aspects tactiques, notamment quand il parle d'un mouvement spécifique: "On fait attention aux déplacements, aux parades et aux esquives. Dans ce cas-là, regarde tu ne peux pas à la fois esquiver et parer. Soit tu bloques et tu rentres mais tu (ne) fais pas une (...) là c'est une réaction de ... c'est un réflexe. Donc tu bloques et tu rentres. C'est à toi de faire ce choix". (SBF-Séance)

Dans cette séance, on observe essentiellement un enseignement tactique par la mise en place d'une opposition. Cela correspond au savoir par la pratique, car les réponses apparaissent en fonction de l'incertitude du combat, que met en œuvre la situation didactique. On observe la transformation du savoir d'expert par le processus de dévolution.

"Riposte, la riposte, donc ce que je veux si je vous demande de travailler en ligne basse, c'est pour pouvoir faire une esquive partielle, c'est à dire (un) déplacement de l'appui, soit à droite soit à gauche donc à vous de trouver la solution." (SBF-Séance)

Il insiste sur le travail tactique en demandant aux étudiants d'utiliser toutes les techniques qu'ils connaissent, selon les mouvements du combat, le rythme, le déplacement, la garde et pendant des situations d'assaut. II leur dit: "à vous de trouver la solution ». Les réponses de l'activité viennent des étudiants, pas du professeur, cela concerne à nouveau le processus de dévolution (Brousseau, 1998) et le savoir par la pratique, dans la mesure où le savoir combattre est rendu accessible aux étudiants par les situations proposées.

Par rapport à l'entretien ante-séance, il semble qu'il a réussi à travailler ce qu'il a proposé et sa méthode insiste surtout sur la technique au service de la tactique. Par conséquent, pour lui, les techniques sont des outils pour être utilisés en réponse aux imprévus du combat. Le savoir combattre qu'il essaie de transmettre est surtout un savoir à s'approprier par la pratique, mais dont les analyses serviront pour la pratique.

5. 2. 2. Analyse de la pratique en escrime de Jean

Jean commence cette séance par un échauffement qui est conduit par un des étudiants et pour lequel il fait quelques corrections par rapport à la biomécanique sous la forme de jogging et d'étirements, sans rapport apparent avec les objectifs de la séance, à l'instar de Roger. Nous observons que les premières vingt-cinq minutes sont organisées sans la mise en place d'un rapport de force, alors que cette variable nous apparaît inséparable de l'enseignement du savoir combattre.

L'enseignant met les étudiants en ligne, pour qu'ils puissent agir selon les signaux du professeur : "Voilà, double marche au signal, près ? Ok, deux retraites après. Oui c'est pas 


\section{eJRIEPS 44 janvier 2019}

mal. Même chose à gauche. Ok, marche et fente! Prêts ? Ok, c'est la première, maintenant petit changement de rythme, marchez, attention à la vitesse à la fin." (Escrime - séance)

Nous pouvons noter qu'il demande aux étudiants une synchronisation des techniques en réalisant les mêmes mouvements que ceux qu'il a lui-même montrés. A ce moment-là, II n'y a pas de rapport de force comme dans la séance de SBF. II insiste sur la répétition décontextualisée des mouvements pendant la première partie du cours. Selon cet enseignant, le plus important est d'automatiser les mouvements d'escrime. La notion de savoir combattre selon les intentions tactiques des adversaires n'est pas mise en scène, car le savoir visé dans ces tâches est plutôt un savoir de la pratique: "Un seul signal. Ce qui m'intéresse, c'est que vous soyez tous synchronisés les uns par rapport aux autres. On doit voir une ligne commencer au bout du match et s'arrêter, une ligne commencer l'attaque et terminer en même temps. A vous de vous synchroniser sur les rythmes des uns par rapport aux autres. Prêts ? Même chose mais je veux voir une seule ligne, ready ?" (Escrime - séance)

Jean explique aux étudiants que le but des exercices est de travailler la coordination, la posture et l'automatisation du déplacement (la technique). Après ces explications, il précise les différents niveaux de défense. A chaque explication, il réunit les étudiants, fait une démonstration lui-même et leur demande de la répéter : “Vous (vous) rapprochez s'il vous plaît ? Vous venez vous asseoir ici, et il me faut une personne pour faire quelques touches avec moi là, qui se dévoue ? Romain, très bien." (Escrime - séance). Nous observons le fait que Jean met des étudiants en opposition, mais sans effectivement induire un rapport de force. Son orientation d'enseignement vise la coopération, dans la mesure où les situations sont peu saturées en incertitude (tâches définies). Même dans le face à face, l'exercice est prévisible, puisque chaque étudiant a un rôle déterminé. "Ok, alors pour commencer, vous allez observer, d'accord, il y aura trois séquences de travail avant d'attaquer les matches, un attaquant, un défenseur. Ton objectif pour toi, ton but, tu donnes le signal puisque tu es le dominant, d'accord on est aux limites du départ, ton objectif à toi c'est de venir me toucher en double marche et fente quand je m'arrête. Vous regardez, je vais vous proposer, il y a cinq niveaux de défense, pour l'instant je vais vous en proposer deux" (Escrime - séance).

Ainsi, la logique de l'escrime appelle t elle un enseignement distinct de la SBF, puisqu'il y a un rôle dominant et un autre dominé règlementés par la phrase d'arme dans le combat. Cela peut justifier le choix du professeur d'enseigner plutôt un savoir orienté vers la 


\section{eJRIEPS 44 janvier 2019}

répétition technique (savoir de la pratique) au lieu d'un savoir par la pratique, avec les éléments d'incertitude, présents dans le rapport de force.

La fin de la séance est organisée sous forme de match : deux étudiants en combat et deux autres en arbitrage (un arbitre principal et un assistant). Le rapport de force est libre, puisqu'il propose les combats formels, selon les règles de l'escrime. Dans ce cas, il manipule la variable didactique de l'arbitrage, en exigeant une posture formelle et sérieuse des étudiants, comme il l'indique dans l'extrait d'échanges entre le professeur et les étudiants : "ce que m'intéresse moi ce ne sont pas les victoires. Par contre ce qui m'intéresse là, c'est que vous travailliez déjà les principes d'attaque, c'est-à-dire la distance, l'attaque en double marche et fente et de temps en temps une attaque en marche et fente, attention, une attaque en marche et fente ». (Escrime - séance).

Dans cette extrait professeur/étudiants, selon le point de vue du professeur, il est important d'avoir un arbitre qui puisse observer la position dominant-dominé dans le combat. Ainsi, le rapport de force au sabre n'est elle jamais libre, puisqu'il a toujours un rôle déterminé à chaque point marqué. Cet aspect sera davantage développé plus tard.

Dans le déroulement de la séance Jean insiste sur les principes d'attaque. Cela apparaît comme une priorité pour lui. II leur demande aussi quelques combinaisons de gestes. II met les étudiants en situation de combat, mais le gain ne l'intéresse pas. Par contre, pour développer les «matches », le professeur insiste surtout sur l'arbitrage des étudiants: « Voilà, au niveau de l'arbitrage : un arbitre, un accesseur, voire deux accesseurs avec les blessés. L'arbitre, ce que j'attends ici, si ça marche bien je corrigerai vos phrases d'armes. » (Escrime - séance).

Nous observons que Jean utilise deux variables didactiques, le rapport de force et l'arbitrage. Les étudiants au cours des matches auront la possibilité de mobiliser le savoir technique appris et le combat lui-même pour faire surgir l'intention tactique, présente seulement dans un enseignement par opposition. Par contre, les étudiants avec le rôle d'arbitrage ont une mission très spécifique, alors qu'ils apprennent les phrases d'armes et la terminologie proposée dans le règlement. En définitive, Jean propose majoritairement des tâches définies (travail de la technique) et semi définies (situation de matches en fin de séance).

Dans son bilan de la séance, Jean observe « qu'au niveau de l'attaque, cela avance » et « qu'au niveau de la défense, cela commence à se mettre en place ». En ce qui concerne le savoir par la pratique (attaquer et défendre), il semble penser que ses étudiants sont sur la bonne voie. 


\section{eJRIEPS 44 janvier 2019}

Toutefois, nous n'avons pas observé une avancée du savoir combattre dans cette séance, II précise encore qu'il leur manque également «beaucoup de choses, notamment de la qualité physique ». Jean signale que, pour lui, il est important d'avoir une bonne qualité physique pour pouvoir exécuter les exercices d'escrime qu'il propose dans ses cours. II complète en disant que dans les prochaines séances, son but sera de travailler la tactique (action-réaction).

\section{Etude comparative des deux cas}

Au regard de notre objet de recherche, qui consiste à analyser le poids de l'expérience sur l'enseignement, nous avons constaté des différences sensibles chez ces deux professeurs. Roger enseigne plutôt des savoirs par la pratique et pour la pratique en stimulant les réponses des étudiants aux problèmes du combat, au travers le processus de la dévolution, de la manipulation des variables didactiques et notamment du rapport de force : « J'essaie de varier au maximum le nombre d'adversaires pour que l'élève s'adapte (...). C'est l'assaut, où là pour moi c'est le retour sur mon enseignement » (Entretien d'après-coup SBF).

Nous pouvons observer une forme caractéristique de transposition didactique chez Roger (SBF), c'est-à-dire qu'il transforme le savoir de la pratique en savoir pour et par la pratique. Les techniques qu'il a enseignées dans les séances antérieures et qu'il a chaque fois corrigées chez les étudiants, correspondent au savoir pour la pratique sous la forme des contenus qu'il a choisi de travailler pour leur formation future.

Les catégories d'analyse que sont le rapport de force et la dévolution ont pour objectif d'introduire l'incertitude, terme qu'utilise d'ailleurs Roger à double reprise dans l'entretien d'après coup. II précise : « Je travaille avec beaucoup plus d'incertitude et c'est à eux de construire ". D'après lui, les étudiants ne sauront combattre que s'ils savent travailler à partir de la notion d'incertitude. "Plus on arrive au bon niveau dans l'activité, plus on arrive à se faire plaisir en adaptant sa boxe à l'adversaire". Le bon niveau selon ce professeur, c'est la capacité de « répondre, échanger, communiquer avec l'adversaire, en y ajoutant à chaque fois beaucoup plus d'incertitude ", c'est «le plaisir à équilibrer le rapport de force, qui est omniprésent ». (Entretien d'après-coup SBF). Pour Roger, enseigner le savoir combattre, c'est introduire l'incertitude à travers des situations de combat, qui comprennent plus d'opposition. Cela concerne le savoir par la pratique, où l'intention tactique émerge des étudiants, au moment du combat, en répondant à sa logique. 


\section{eJRIEPS 44 janvier 2019}

Jean enseigne plutôt des savoirs de la pratique et pour la pratique, c'est-à-dire qu'il met l'accent sur la reproduction des techniques décontextualisées, sur l'arbitrage formel et il utilise des situations d'opposition avec des rôles déterminés.

Cette forme de transposition s'observe plus par des transformations des contenus didactiques sous la forme de répétition des mouvements et un peu moins par la manipulation du rapport de force. II enseigne les savoirs de l'escrime dans la séance, mais il ne semble pas enseigner l'essence du savoir combattre, soit le rapport de force. Dans l'entretien d'après-coup, en caractérisant sa démarche, il précise que dans son cycle il associe la connaissance des disciplines biologiques avec une connaissance qu'il appelle globale et qui n'est pas seulement technique, comme peut l'être traditionnellement l'escrime. Son intention semble donc de réunir les connaissances biologiques, la pédagogie et la didactique. «Je suis sur une méthode globale et je fais des apprentissages concernés nécessaires pour la pédagogie et la didactique ». (Entretien d'après-coup escrime).

Le discours de Jean sur le cycle est différent de ce qu'il fait dans la séance observée. Dans la séance que nous avons analysée, Jean nous dit que les étudiants ne possèdent pas les qualités physiques pour exécuter les exercices. Les éléments biologiques sont très importants pour lui (quand il dit par exemple qu'il manque des « qualités physiques chez les étudiants pour exécuter les activités»). Les apprentissages vont plutôt vers l'automatisation et la répétition des techniques. Pendant la séance, les techniques ont été décontextualisées, alors qu'en opposition, le principe de l'incertitude est peu développé, contrairement à Roger.

Dans le cours de Jean que nous avons observé, les étudiants ont été mis dans des tâches décontextualisées en réponse aux commandes du professeur et en duo avec des rôles déterminés. Dans d'autres tâches comme, pendant les matches, Jean a mis davantage l'accent sur l'arbitrage : «Si je n'ai pas d'arbitre, je ne peux pas avoir de matches » et sur la priorité d'attaque de chaque combattant : «Donc le but pour le dominé, c'est d'essayer de trouver un moyen pour renverser le rapport dominant-dominé ». (Entretien d'aprèscoup escrime)

Quand nous interrogeons Roger sur les raisons de son choix du sabre, il confirme l'hypothèse que son histoire personnelle - particulièrement son déjà-là expérientiel - a influencé sa conception d'enseignement. « Oui, que le sabre. Pourquoi ? Parce que moi je suis sabreur déjà à la base et c'est l'arme la plus riche et la plus facile pour démarrer et s'amuser ». (Entretien d'après-coup escrime). 


\section{eJRIEPS 44 janvier 2019}

Donc, dans cette Université, Jean n'enseigne que le sabre. Au début de la recherche, nous pensions que c'était parce qu'il considérait le sabre plus riche. En fait, il explique pendant l'entretien d'après-coup qu'il enseigne seulement le sabre parce qu'il a été un sabreur d'un haut niveau, donnée qui conforte notre hypothèse. II semble alors que l'expérience personnelle du professeur est directement liée aux contenus de l'APSA qu'il transmet dans ce cas précis, en rapport avec la notion de conversion didactique développé dans l'article (Cf : paragraphe concerné dans l'article).

Cette étude fait également émerger des différences entre technique et tactique entre les deux enseignants qui justifient l'utilisation qu'ils en font. Alors que pour Jean la technique est transmise comme élément fondamental de l'activité et qu'il appartient à l'étudiant de gérer lui-même la situation d'opposition, pour Roger, la technique est le plus souvent transmise dans une situation d'affrontement comme moyen de résoudre, in situ, le rapport de force entre deux opposants. Ainsi, parade et riposte peuvent être envisagées séparément, sans opposition, ou bien transmises comme indispensables à l'établissement du rapport de force.

\section{Analyse des résultats : les raisons présumées des différences constatées}

7. 1. La différence de trajectoire et de formation

Roger nous informe avoir appris cette activité à l'Université et n'a pas de passé de compétiteur. II essaie de transmettre un savoir significatif pour que ses étudiants puissent avoir un ensemble d'éléments de SBF leur permettant d'enseigner eux-mêmes cette activité en EPS et peut être dans les clubs. « Je n'ai pas une vision sportive de haut niveau de l'activité (...) C'est vrai que j'ai un regard qui n'est pas uniquement axé sur un passé de combattant qui est chez moi assez minime ». (Entretien d'après-coup SBF).

Jean a été lui aussi professeur d'EPS en même temps qu'il a été compétiteur (champion olympique de sabre en équipe). II a construit sa méthode en utilisant ce qu'il a vécu comme enseignant, mais aussi et surtout comme sportif de haut niveau en tant que sabreur. Nous faisons l'hypothèse que sa conception garde beaucoup de la tradition très ancienne de l'activité et qu'il essaie plutôt de transmettre un savoir corporel orienté vers l'exécution des techniques d'escrime qu'un savoir enseignable à d'autres. « Oui, j'ai trouvé un sens de pourquoi m'entraîner. Ça vient du haut niveau, de la performance » (Entretien d'après-coup escrime). 


\section{eJRIEPS 44 janvier 2019}

7. 2. L'escrime et la Savate Boxe Française : deux activités physiques différentes La spécificité de chaque activité peut également influencer le traitement didactique de ces professeurs. En effet, la dynamique de la SBF est différente de l'escrime, et dans l'escrime il a encore des différences entre l'épée, le fleuret et le sabre.

Jean est un sabreur et sa pratique ne concerne que les éléments du sabre. Dans la convention du sabre, le tireur qui touche en premier n'est pas obligatoirement celui qui emporte le point (Capoani, d'Ares, 2010). La touche se donne selon un principe de priorité. Le tireur qui exécute correctement l'attaque (c'est-à-dire l'action offensive initiale) a la priorité sur toute autre action et emporte le point. Le tireur attaqué n'a d'autre solution que de parer l'attaque et de riposter ou de profiter d'une mauvaise exécution de l'attaque pour reprendre la priorité (Capoani, d'Ares, 2010). Cette dynamique est notamment distincte de la logique du rapport de force, dans la mesure où il n'y a pas d'actions d'attaque et défense simultanément. Pour respecter les principes du sabre, Jean peut se trouver limité pour proposer un rapport de force aux étudiants, puisque dans les règles du sabre, il manque un principe conditionnel (l'attaque-défense simultanée)

Le sabre (comme le fleuret) peut être considéré comme une activité moins imprévue que l'épée où il n'y a pas ce type de priorité, ce qui explique les raisons pour lesquelles le professeur d'escrime préserve surtout le savoir de la pratique, compte tenu des risques encourus.

La SBF, par contre, répond à tous les principes conditionnels (Gomes, 2008) et notamment l'attaque et défense simultanées puis l'incertitude. Cela donne aux professeurs la possibilité de manipuler un rapport de force et d'autres variables didactiques et d'enseigner le savoir combattre en stimulant l'intention tactique, tel que proposé par Terrisse et al (1995). L'absence d'une arme permet une diversité de choix didactiques concernant l'espace, les matériels, la distance, les coups de poings, des pieds et la logique du combat de la SBF.

Dans cette recherche, l'analyse didactique effectuée ne peut faire l'impasse sur la différence de nature de ces deux activités.

\section{Conclusions}

L'analyse des résultats a montré une grande différence d'enseignement chez ces deux professeurs: Roger (SBF) et Jean (escrime). Les choix et les intentions didactiques de chaque professeur ont été entendus sous la forme d'entretien ante-séance en reconstruisant leurs intentions didactiques relatives au savoir à enseigner. Cela nous a 


\section{eJRIEPS 44 janvier 2019}

permis d'observer que chaque professeur fait ses choix didactiques par rapport à ce qu'il a vécu, soit comme combattant, soit comme étudiant en STAPS (sa formation). C'est de cette manière que le savoir de la pratique est incorporé par chacun.

Pendant les enregistrements vidéo et audio des séances, nous avons observé le savoir enseigné, au moment de l'épreuve, où le professeur met en place son choix, pour rendre enseignable le savoir de la pratique aux étudiants au travers d'un processus de “ conversion didactique " (Buznic, 2009). A cette étape-là, nous avons constaté que le passé d'étudiant en STAPS de Roger (SBF), comme la nature de l'activité de la SBF l'ont induit, plus ou moins consciemment, à construire une stratégie d'enseignement principalement orientée vers un savoir par la pratique et pour la pratique, vers l'appropriation du savoir combattre, à travers de l'opposition.

De manière contrastée, Jean, au travers de la séance analysée, a montré que même en ayant l'intention d'une stratégie plus globale et moins guidée vers la pure technique, son passé de compétiteur et surtout la nature de l'arme (le sabre), l'amènent plus ou moins à son insu à transmettre un savoir de la pratique pour la pratique, enseigné sur le même modèle que celui qui lui a été enseigné (Brau-Anthony, 1998).

Les catégories d'analyse ont été des outils pour rendre compte de l'enseignement et contribuent à la compréhension de la conversion didactique à l'œuvre dans les deux enseignements. Dans les entretiens d'après-coup, la possibilité de revenir à certains moments sur l'enseignement, nous a permis de confirmer nos hypothèses et de confronter les intentions des professeurs avec ce qu'ils ont réellement enseigné.

L'analyse de pratiques d'enseignement des Sports de Combat dans cette Université indique qu'en STAPS, ces professeurs de SBF et d'escrime enseignent le savoir combattre de façon différente, selon les spécificités de chacun (leur formation, leurs conceptions de l'APSA, leurs expériences personnelles et professionnelles, leurs intentions) et de chaque activité physique enseignée, alors qu'ils sont dans la même institution et qu'ils ont les mêmes étudiants. Cette étude souligne la pertinence d'un regard clinique en didactique de l'EPS qui - au-delà des assujettissements institutionnels, officiels et personnels (Chevallard, 1989) - repose sur la prise en compte de la singularité des sujets et sur l'influence de leur trajectoire personnelle sur leurs choix didactiques. 


\section{eJRIEPS 44 janvier 2019}

\section{Bibliographie}

Bardin, L. (1998). L'analyse de contenu. Paris : PUF. (9ème édition).

Ben Jomaa, H. (2009). L'expertise dans l'enseignement de l'EPS : analyse didactique clinique de quatre professeurs. Thèse de doctorat en cotutelle. UPS, Toulouse 3 et Université de La Manouba (Tunisie).

Brau-Anthony, S. (1998). L'évaluation des jeux sportifs collectifs. Des conceptions des enseignants d'EPS à la démarche de mise en œuvre du projet d'enseignement et d'évaluation. Thèse de doctorat non publiée. Université Paris 11.

Brossais, E. \& Terrisse, A. (2009). Le poids de l'institution sportive dans l'analyse d'un enseignant débutant - expert en judo. Dans A. Terrisse \& M-F. Carnus (Dir.), Didactique clinique de l'EPS : quels enjeux de savoirs ? (p. 115-132). Bruxelles : De Boeck Université.

Brousseau, G. (1998). Fondements et méthodes de la didactique. Dans B. Balacheff, M. Cooper, R. Sutherland, \& V. Warfield (Dir.), Théorisation des situations didactiques (p. 47-10). Grenoble : La Pensée Sauvage,

Bru, M. (1991). Les variations didactiques dans l'organisation des conditions d'apprentissage. Toulouse : Presses Universitaires du Sud.

Buznic, P. (2009). Le sujet enseignant et la gestion du contrat didactique. Un regard en didactique clinique de l'EPS. Dans A. Terrisse \& M-F. Carnus (Dir.). Didactique clinique de l'EPS: quels enjeux de savoirs ? (p. 101-112). Bruxelles : De Boeck Université,

Capoani, J., d'Ares, J.C.D. (2010). Les fondamentaux de l'escrime : Fleuret, épée, sabre : S'initier et progresser. Paris : Amphora.

Carnus, M.-F. (2001). Analyse didactique du processus décisionnel de l'enseignant d'EPS. Thèse non publiée, LEMME, Université Paul Sabatier, Toulouse

Carnus, M-F. (2004). La prise en compte du sujet dans la recherche en didactique, vers une didactique clinique : intérêt, difficultés et limite. Dans symposium : Recherche clinique en Sciences de l'Éducation, Contribution pour une analyse des pratiques enseignantes, Cinquième congrès international de l'AECSE d'actualité de la recherche, 31 Août, 4 Septembre 2004, Paris, CNAM.

Carnus, M-F. (2009a). Pour une didactique clinique de l'EPS. Perspectives pour la formation des enseignants. Note de synthèse pour l'Habilitation à Diriger des Recherches. Université Paris Ouest Nanterre la Défense, 9 décembre 2009. 


\section{eJRIEPS 44 janvier 2019}

Carnus, M.-F. (2009b). La décision de l'enseignant en didactique clinique. Etudes de cas en Education Physique et Sportive. Dans A. Terrisse et MF Carnus (dir.), Didactique clinique de l'EPS, Quels enjeux de savoirs ? (p. 63-81). Bruxelles : De Boëck.

Carnus M-F. \& Terrisse A. (2013). Didactique clinique de l'EPS. Le sujet enseignant en question. Paris : Editions revue EPS.

Chevallard, Y. (1985). La transposition didactique. Grenoble : La Pensée Sauvage.

Dorville, C. (2004). Des pieds et des poings : la boxe française. La nouvelle jeunesse d'un vieux sport français. Villeneuve d'Ascq : Revue du Nord, vol. 86(355).

Famose, J-P. (1983).Tâches motrices et stratégies pédagogiques en éducation physique et sportive - Dossiers EPS n`1. Revue EP\&S. Paris.

Gomes, M.S.P. (2008). Procedimentos pedagógicos para o ensino das lutas: contextos e possibilidades. 119f. Dissertação (Mestrado em Educação Física) - Faculdade de Educação Física Universidade Estadual de Campinas, Campinas.

Gomes, M.S.P. Morato, M. Duarte, E., Almeida, J.J.G. (2010). O Ensino das Lutas: dos princípios condicionais aos grupos situacionais. Movimento, Porto Alegre, v.16, n.02, 207-227, abr-jun.

Heuser, F. (2009). Du savoir enseigné en EPS au savoir de référence de l'enseignant. Etude didactique clinique en karaté. dans A. Terrisse \& M-F. Carnus (Dir.). Didactique clinique de l'EPS : quels enjeux de savoirs ? (p. 133-142). Bruxelles : De Boeck Université.

Loizon, D., Margnes, E., Terrisse, A., (2004). Analyse des pratiques d'enseignement : les pratiques déclarées par les professeurs de judo. eJRIEPS, 5, 63-85.

Margnes E. (1996). Réflexions sur les valeurs du judo et perspectives de transposition didactique en EPS, dans Arts martiaux et Sports de combat, les cahiers de I'INSEP, Paris, INSEP Publications, 173-179.

Reuter Y. (éd.) (2007). Dictionnaire des concepts fondamentaux des didactiques, Bruxelles : De Boeck.

Sauvegrain, J-P. (2001). Analyse didactiques de la décision de l'élève, étude de cas sur l'utilisation du savoir dans un cycle de lutte. Thèse de doctorat. Université Paul Sabatier. Toulouse.

Green A. \& Svinth J-R (2010). Martial Arts of the Words. An Encyclopedia of History and innovation. ABC - CLIO. EBook 978-1-59884-244-9. June 2010. Vol. 2. 
eJRIEPS 44 janvier 2019

Terrisse, A. (1991). Pour un enseignement dialectique du combat. Revue E.P.S., 229, 2326.

Terrisse, A. (1994). La question du savoir dans la didactique des Activités Physiques et Sportives : essai de formulation. Note de synthèse pour l'HDR. Université Paul Sabatier. Toulouse.

Terrisse, A., Quesada, Y., Hiegel, P., Sauvegrain, J.P. (1995) : Le savoir combattre : essai d'élucidation, Revue E.P.S., 252, 26-29.

Terrisse, A. \& Sauvegrain, J. P. (1998). Identification des difficultés d'appropriation du "savoir lutter » par une méthodologie d'ingénierie didactique. Revue Science et Motricité, 32-33, 74-87.

Terrisse, A. (2000). Epistémologie de la recherche clinique en sports de combat. Dans Terrisse, A., (Dir.). Recherches en sports de combat et en Arts Martiaux, état des lieux (p. 95-108). Paris : Editions Revue EPS.

Terrisse, A. (2009). La didactique clinique en EPS. Origine, cadre théorique et recherches empiriques. Dans A. Terrisse \& M-F. Carnus (Dir.). Didactique clinique de l'EPS: quels enjeux de savoirs? (p.13-33). Bruxelles : De Boeck Université.

Terrisse, A. \& Carnus, M.-F. (2009). Didactique clinique de l'EPS: quels enjeux de savoirs ? Bruxelles : De Boeck Université.

Thomas, J. R \& Nelson, J. K. (2002). Métodos de pesquisa em atividade física. Porto Alegre : Artmed.

Verret, M. (1975). Le temps des Etudes. Paris : H. Champion. 